\title{
Primitive Neuroectodermal Tumor
}

National Cancer Institute

\section{Source}

National Cancer Institute. Primitive Neuroectodermal Tumor. NCI Thesaurus. Code C3716.

A malignant neoplasm that originates in the neuroectoderm. The neuroectoderm constitutes the portion of the ectoderm of the early embryo that gives rise to the central and peripheral nervous systems and includes some glial cell precursors. 